\title{
La relevancia de la confianza en conflictos socioambientales por energía en Chile: los casos de "Castilla" $\mathrm{e}$ "Hidroaysén"1
}

\author{
Arturo Vallejos-Romero², Alex Boso ${ }^{3}$ y Hugo Marcelo Zunino 4
}

\begin{abstract}
RESUMEN
La dinámica social moderna ha generado riesgos y peligros, situación que se visibiliza en conflictos socioambientales que emergen en distintos contextos socioespaciales, representando las tensiones existentes entre intereses contrapuestos. Una de las dimensiones sociales relevada por la literatura para gobernar los riesgos que genera la conflictividad socioambiental es la confianza, variable que modelos y diseños de intervención han tomado poco en cuenta. A partir de una investigación exploratoria integrada se levantó información a través de 6 grupos de discusión y 15 entrevistas semiestructuradas para analizar la confianza, con el objetivo de evidenciar su incidencia en las relaciones entre actores sociales e instituciones públicas y privadas en los conflictos Termoeléctrica Castilla e Hidroeléctrica Hidroaysén en Chile. Los resultados destacan que Competencia-Habilidad y Consistencia del Comportamiento (variables objetivas), así como Benevolencia, Integridad, Sentido de Justicia y Cumplimiento de los Compromisos (variables subjetivas), explican las relaciones, tensiones y salidas en los conflictos analizados.
\end{abstract}

Palabras clave: Riesgos, Peligros, Participación, Capacidades Institucionales.

\begin{abstract}
Modern social dynamics are related to increasing hazards and risks, a situation that is crystalized in socio-environmental conflicts that arise under different socio-spatial contexts, representing the existing tensions among contrasting interests. One of the social dimensions reported in the literature on the governance of risks that generate socio-environmental conflicts is trust, a variable that intervention models have not been able to account for in a satisfactory manner. Based on exploratory research using an integrated design, which included 6 focus groups and 15 semi-structured interviews, we examine the impact of trust on the relationship between social actors and public and private institutions in the conflicts over the proposed thermoelectric (Castilla) and hydroelectric (Hidroaysén) projects in Chile. The results highlight that competence and behavioral consistency (objective variables), along with compassion, integrity, a sense of justice and the compliance with promises (subjective variables) explain the relationships, tensions, conflicts and conflict resolution in the cases analyzed.
\end{abstract}

Key Words: Risk, Hazards, Civic Participation, Institutional Capacity, Chile.

1 Este artículo es producto del Proyecto Fondecyt $\mathrm{N}^{\circ}$ 1120554. Artículo recibido el 11 de diciembre de 2014, aceptado el 28 de mayo de 2015 y corregido el 31 de agosto de 2015.

2 Núcleo de Investigación en Ciencias Sociales y Humanidades, Universidad de La Frontera (Chile). E-mail: arturo.vallejos@ufrontera.cl

\footnotetext{
3 Núcleo de Investigación en Ciencias Sociales y Humanidades, Universidad de La Frontera (Chile). E-mail: alex.boso@ufrontera.cl

4 Departamento de Ciencias Sociales, Núcleo de Investigación en Ciencias Sociales y Humanidades Universidad de La Frontera (Chile). E-mail: hugo.zunino@ufrontera.cl
} 
La sociedad del riesgo y las políticas de desarrollo han generado un aumento de la preocupación pública sobre los problemas socioambientales provocados por proyectos energéticos.En esta dinámica, los riesgos y peligros generados han tenido repercusiones globales (Giddens, 1993, Beck, 2008, 2006, 2002; Luhmann, 2007, 2006) que se presentan de un modo imprevisible $y$, hasta cierto punto, inevitables. Dicho de otro modo, los riesgos derivados de proyectos de desarrollo energéticos están desarticulando los formatos establecidos de seguridad y afectando a modernidades periféricas como América Latina, donde los mecanismos para prevenir y controlar las amenazas generadas por las tecnologías se muestran cada vez más ineficientes.

Junto a la creciente preocupación pública sobre los riesgos tecnológicos de instalaciones energéticas, se suele observar una reducción de la confianza en el gobierno y en los responsables de la industria, así como un incremento de esta en las asociaciones cívicas $\mathrm{u}$ organizaciones ambientalistas (Peters et al., 1997). En cualquier caso, la confianza es identificada en el debate académico como un elemento clave de la percepción pública de los riesgos tecnológicos (Reen y Levine, 1991; Pidgeon et al., 1992; Poortinga y Pidgeon, 2003; Terwel et al., 2009; Oltra et al., 2014; Upham et al., 2015). En general, la literatura tiende a considerar que la efectividad de la comunicación y el manejo de los riesgos depende de la confianza y, a su vez, ambos elementos son capitales para lograr una adecuada adaptación a los peligros inherentes a las instalaciones energéticas (Kasperson et al., 1992; Solá et al., 2009; Visschers et al., 2011).

En Chile, diversos trabajos han alertado sobre los crecientes conflictos socioambientales (Cárcamo et al., 2011; Romero-Toledo, 2014; Romero-Toledo et al., 2009, 2012; Rojas et al., 2003). En este estudio se analiza la relevancia que cobra la confianza (interpersonal e institucional) en la gestión de los riesgos asociados a las instalaciones de producción de energía en Chile, tomando los casos de la Termoeléctrica Castilla en la Región de Atacama (de ahora en adelante Castilla) e Hidroeléctrica Hidroaysén en la Región de Aysén (de ahora en adelante Hidroaysén).

\section{Los riesgos y la conflictividad socioambiental}

Los estudios y definiciones sobre riesgos ha sido amplios y no menos ambiguos. El clásico economista Frank Knight (2013) realiza una distinción entre riesgo e incertidumbre. Para él, una situación riesgosa puede darse cuando variadas acciones llevan a distintos resultados, conociéndose la probabilidad de todos ellos, mientras que hay incertidumbre cuando las probabilidades de sus resultados no se conocen. Bajo estas premisas, se puede decir que en las afirmaciones de Knight existe objetividad, en el sentido que cada suceso tiene la probabilidad cierta y objetiva de ser conocido, lo cual la literatura ha puesto desde algún tiempo en entredicho (Douglas, 1986; O'Malley, 2002, 2005; Rosa et al., 2013; Zinn, 2008; Mairal, 2013).

En este estudio nos apartamos de la mirada de Night y tomamos la distinción riesgo/ peligro (Luhmann, 2006, 2007), la cual apunta a una externalidad o daño futuro; por lo tanto incierto. Para esta línea, si la externalidad está asociada a una decisión será un riego, y si el daño está ligado a una acción del entorno, será un peligro (Luhmann, 2006). En consecuencia, riesgo y peligro dependerá de quién observe, pues -por ejemplo- las externalidades o daños por la instalación de una termoeléctrica o hidroeléctrica se pudieron haber evitado si se hubiera decidido no instalarlas (lo cual es un riesgo). Por otro lado, los daños causados por una crecida de un río a causa de una erupción volcánica pueden ser eventos del entorno (peligros), pero si se hubiera decidido no poner casas en lugares inapropiados, estos no hubieran sucedido (riesgos).

Los riesgos en torno a conflictos socioambientales ${ }^{5}$, especialmente los ligados a ener-

\footnotetext{
5 Un conflicto socioambiental es una contradicción o controversia de información, intereses o valores entre actores (personas, grupos, organizaciones) que se genera en la sociedad y refiere a cuestiones de acceso, disponibilidad y calidad de los recursos naturales como construidos, así como de las condiciones sociales y ambientales del entorno que afectan la calidad de vida de la sociedad (Fiske, 2000; Vallejos, 2005).
} 
gía y generados por termoeléctricas en Chile, pueden ser observados y atribuidos, primariamiente, como resultado de la producción y reproducción de la sociedad moderna, y secundariamente, como producto del diseño e implementación de los modelos de desarrollo económico, anclados en pujantes procesos de industrialización, urbanización y grandes megaproyectos que han tenido lugar en las últimas décadas (Altieri y Rojas 1999; Quiroga 2001). Los problemas y riesgos socioambientales derivados de este proceso, no solo se han expandido y complejizado en las últimas décadas (Camus y Hajek 1998), sino que la capacidad privada e institucional para prevenirlos y resolverlos parece haberse visto sobrepasada (OCDE, 2005; Sepúlveda y Rojas, 2010).

Ante la percepción generada por el advenimiento de nuevos riesgos socioambientales, la sociedad ha reaccionado a través de una conflictividad que ha escalado en intensidad (Fiske, 2000). Sin embargo, los conflictos no se han manifestado como respuestas automáticas, sino que han sido mediatizados por el significado que las organizaciones y actores sociales atribuyen a los problemas socioambientales (Beck, 1995; Sepúlveda y Geisse, 1995). Por un lado, la expresión social ante el aumento de los riesgos y sus externalidades da lugar a conflictos de una gran reverberancia social. Por otro, los problemas socioambientales también se multiplican sin que necesariamente generen manifestaciones explícitas, lo que no quiere decir que no sean visibilizados o percibidos por quienes son impactados (Espluga, 2004, 2007; Espluga y Ferré, 2007).

Los conflictos y riesgos socioambientales derivados de las instalaciones energéticas son instancias privilegiadas para observar las reacciones y respuestas sociales ante las externalidades socioambientales que estas producen. Mediante su observación puede analizarse la configuración y perfil de los actores que se confrontan, la relación que se da entre estos, así como sus percepciones sobre los problemas y conflictos socioambientales.

Explorar la problemática planteada es relevante para la gestión de los riesgos, pues Chile en la actualidad ha tomado y tomará decisiones en torno a su matriz energética, donde la principal fuerza de esta sería la fuente hidráulica (agua) y termoeléctrica (carbón y petróleo) (Aguilar y Valenzuela, 2010; AA.VV, 2010). La tensión social generada por los conflictos en torno a la producción de energía podría ser profunda. En este contexto, entender cabalmente los mecanismos sociales que operan entre la confianza y la gestión efectiva de los riesgos es una tarea indispensable para generar conocimiento en torno a un incipiente campo interdisciplinar en Chile.

\section{La confianza y su relevancia en los conflictos socioambientales}

En términos amplios, la confianza puede ser entendida como un dispositivo ordenador y reductor de complejidad de la convivencia social. Por consiguiente, constituye un recurso para cualquier acción a futuro que puede probabilizar comportamientos y otorgar ciertas garantías de seguiridad. Cuando este recurso se pierde, tienden a cuestionarse las acciones y capacidades personales e institucionales, así como las políticas diseñadas para el manejo de los riesgos y peligros. Por consiguiente, los procesos de pérdida de confianza tienden a visibilizarse en conflictos socioambientales como los termoeléctricos en Chile, entre otros.

En este contexto, los estudios del riesgo han comenzado a prestar una fuerte atención a la confianza, que funge como "un prerrequisito para conseguir una efectiva comunicación y gobernanza del riesgo" (Kasperson et al., 1992, citado por Espluga et al., 2009: 3). La confianza, como un mecanismo de reducción de complejidad (Luhmann, 1996), se torna una variable esencial que favorece la emergencia de actuaciones coherentes de las instituciones y público en un entorno diverso y en ocasiones desbordante. Ante situaciones de incertidumbre y asimetrías informativas, la confianza establece certeza interna que garantiza cada una de las operaciones en el momento de tomar decisiones.

A pesar de que existe un acuerdo general en la literatura académica sobre la importancia de la confianza, los científicos sociales han mostrado ciertas discrepancias acerca de cómo conceptualizarla y estudiarla (Chryssochoidis et al., 2009). Espluga et al. 
(2009), Rodríguez y Majluf (2003), Majluf et al. (2003) y Rodríguez (2009) exponen profusamente los esfuerzos particulares de conceptualizar la confianza interpersonal e instuticional desde la sociología, la psicología y las ciencias políticas. Algunos autores argumentan que la confianza debe ser descrita mediante dos factores fundamentales como son la competencia (o grado de conocimiento y experiencia técnica percibido) y la afección (Eiser et al., 2009; Espluga 2009). En este sentido, Espluga enmarca la confianza como un constructo bidimensional con un ámbito técnico como la competencia, la capacidad, la eficiencia, la consistencia, el conocimiento y experiencia experta, así como una dimensión afectiva, donde releva dimensiones como la objetividad/imparcialidad, la honradez, la buena voluntad, la preocupación por la salud y el bien común, la integridad y el compromiso con los intereses de los demás.

Rodríguez y Majluf (2003) elaboran para Chile un modelo para medir confianza interpersonal (ya sea esta directa o indirecta), donde proponen un tipo de confianza basada en la razón y otro basado en el afecto. Para el primero (razón), los autores identifican componentes como la competencia o habilidad, la benevolencia y la integridad, sentido de justicia, cumplimiento de compromisos y consistencia del comportamiento, mientras que para el segundo tipo (afecto) reconocen dos elementos, la apertura comunicacional y la ausencia de conflicto. Esta operacionalización permite ordenar las dimensiones técnicas y afectivas propuestas por Espluga et al. (2009), lo cual nos acerca a un modelo orientador para dar cuenta de las pretensiones de nuestra trabajo.

Bulloch (2013) señala que desde un punto de vista teórico-conceptual la confianza tiene dos vertientes: una moral y otra estratégica. Nuestra aproximación se aparta de la primera vertiente, que concibe la confianza como una creencia en la bondad de la naturaleza humana en general (Yamagishi y Yamagishi, 1994, citado por Bulloch, 2013). En este estudio se analiza la visión estratégica de la confianza donde: i) los individuos confían porque es razonable hacerlo; ii) la confianza será posible siempre que tenga algún depositario; y iii) la información y la experiencia constituyen elementos clave. La confianza, en su vertiente estratégica, funge como cemento social, ya que es necesaria para accionar diariamente sin preguntarnos sobre las formas que la sociedad ha estabilizado. Por otro lado, la confianza es una reductora de la complejidad social y puede ser observada, testeada y medida a través de variables e indicadores que puedan dar cuenta de sus rendimientos en una sociedad moderna, especialmente -para nuestro caso- en conflictos socioambientales en torno a energía en Chile (Vallejos, 2012, 2015).

Anclados en lo anterior, nos desprendemos de una observación moral, normativa y de control y nos hacemos cargo de una mirada de invitación u orientación a confiar en las acciones institucionales sobre los riesgos, donde las estrategias de intervención o gestión deben estar acorde a la complejidad social. En este sentido, es relevante indagar en el perfil y las relaciones de confianza entre los actores implícitos en conflictos socioambientales en torno a energía como Castilla e Hidroyasén. En lo operativo, se hace necesario observar dimensiones objetivas y subjetivas de la confianza en las instituciones y relaciones interpersonales como las que se presentan el Cuadro $\mathrm{N}^{\circ} 1$. 
Cuadro $\mathrm{N}^{\circ} 1$

Dimensiones generales y específicas para la percepción de confianza

\begin{tabular}{|c|c|c|}
\hline & \multicolumn{2}{|c|}{ Dimensiones Específicas } \\
\hline Dimensiones Generales & Competencia Técnica & Afectiva \\
\hline Competencia/Habilidad & $\begin{array}{l}\text {-Experiencia (Qué han realizado) } \\
\text {-Eficiencia (Cómo lo han realizado) } \\
\text {-Capacidades (Recursos financieros, téc- } \\
\text { nicos, humanos e infraestructura) } \\
\text {-Competencias (técnicas como legales) }\end{array}$ & \\
\hline Benevolencia & & $\begin{array}{l}\text {-Preocupación por la } \\
\text { salud y el bien común } \\
\text {-Buena Voluntad. }\end{array}$ \\
\hline Integridad & & $\begin{array}{l}\text {-Honradez } \\
\text {-Transparencia }\end{array}$ \\
\hline Sentido de Justicia & & $\begin{array}{l}\text {-Objetividad/Impar- } \\
\text { cialidad }\end{array}$ \\
\hline $\begin{array}{l}\text { Cumplimiento de compromi- } \\
\text { sos }\end{array}$ & & $\begin{array}{l}\text {-Compromiso con } \\
\text { intereses de los demás }\end{array}$ \\
\hline Consistencia comportamiento & $\begin{array}{l}\text {-Consistencia /predictibilidad de com- } \\
\text { portamientos y argumentos }\end{array}$ & \\
\hline
\end{tabular}

Fuente: Elaboración propia en base a Rodríguez y Majluf( 2003) y Espluga et al. (2009).

\section{Los casos termoeléctrica Castilla e Hidroaysén}

El proyecto Termoeléctrica Castilla (20082010) pretendía instalar una Central Termoeléctrica a Petróleo Diésel y una Central Termoeléctrica a Carbón, la cual pasaría a ser una de las mayores centrales de producción de energía en Chile y la mayor central a carbón en Sudamérica. Los montos de inversión fluctuaban en los UM\$4.400, uno de los más altos en el país. La propuesta de instalación generó una alta connotación social, tanto a escala nacional como internacional, desencadenando un conflicto que movilizó y enfrentó a distintos actores de la comunidad local y nacional. Por un lado, se articularon las posiciones de apoyo que señalaron que el proyecto era necesario en el entendido "que las energías limpias, solo pueden cubrir una parte de la demanda y el resto del abastecimiento deberá ser siempre desarrollado por energías hidroeléctricas y térmicas" (Vilches, 2010). Dichas posiciones exponían que, de acuerdo a la normativa local, la propuesta estaba resguardada dado que el plan regulador definía un uso industrial para la zona. Por otro lado, la oposición señalaba los elevados daños que ocasionaría el proyecto al ecosistema de la zona, aduciendo que "para poder enfriar los generadores se sacará agua del mar la que será devuelta con aproximadamente $8^{\circ} \mathrm{C}$ más, lo que provocaría la muerte de peces y mariscos del sector, además de dejar sin trabajo a muchas familias de pescadores de la zona" (Giro País, 25-02-2011). Siendo el carbón contaminante y tóxico, con altas externalidades negativas al medio natural y humano, el proyecto pone en tensión el desarrollo, los ejes de la matriz energética, las decisiones que se toman para su implementación (altamente cuestionadas) y los impactos a la calidad de vida y salud de las comunidades, así como el impacto a las actividades de los pescadores artesanales y el patrimonio del Totoral ${ }^{6}$.

\footnotetext{
6 Zona de pescadores artesanales ubicadas en la Tercera Región de Atacama, Chile.
} 
El proyecto Hidroaysén se emplazaría en las comunas de Cochrane, Tortel y O’Higgins, en la Región de Aysén, y consistiría en la construcción de un Complejo compuesto por cinco centrales de generación (Baker 1, Baker 2, Pascua 1, Pascua 2.1 y Pascua 2.2). Una vez operativo el complejo, contaría con una potencia instalada de 2.750 MW y generaría una energía media anual de 18.430GWh, que será incorporada al Sistema Interconectado Central (SIC) (SEIA, 2008). Las empresas inversionistas serían Endesa y Colbún e invertirían un monto de UM\$ 3.200. La argumentación a favor del proyecto era que Chile debía tener una mayor independencia y seguridad energética mediante el aprovechamiento sustentable de un recurso propio, limpio, renovable y estratégico como es el agua. Asimismo, el proyecto dinamizaría la economía y potenciaría las condiciones para el desarrollo social de la región de Aysén, incorporando mayor conectividad al mejorar infraestructura asociada al transporte terrestre, aumentando el empleo y favoreciendo el desarrollo de sus habitantes (SEIA, 2008). Quienes lo rechazaban aducían graves daños a la integridad ambiental de un valioso territorio, cuya crítica apuntaba a una escasa voluntad de políticas por energías limpias que podrían satisfacer la demanda nacional, a una visión de futuro poco clara y a un modelo de desarrollo que provocaría grandes perjuicios al entorno natural. Por tanto, Hydroaysén sería, para las voces críticas, un proyecto " tremendo" (New York Time, 24-05-2011) y un " error irresponsable" (El Mostrador, 24-05-2011).

\section{Metodología}

El artículo está enmarcado en una propuesta de investigación mayor, de tipo exploratoria e integrada, la cual tuvo como objetivo caracterizar y explicar las relaciones de confianza y comunicación que se dan entre instituciones públicas, privadas y los actores sociales en torno a los riesgos que generan los conflictos socioambientales por energía en Chile. El diseño fue el Estudio de Casos, para el cual se elaboraron y aplicaron tres instrumentos: una encuesta, seis grupos de discusión y quince entrevistas semiestructuradas a actores claves en los conflictos descritos. En el presente artículo, se analiza la información cualitativa resultante de los grupos de discusión y las entrevistas semiestructuradas para los dos conflictos mencionados, con el objetivo de examinar las construcciones y percepciones de riesgos que los actores en ambas localidades (Atacama y Aysén) "... enfrentan de manera cotidiana" (Tejeda y Pérez-Floriano, 2011: 84), ayudando a entender las "estructuras complejas de identidad y su contexto social" (Smith et al., 2006; Zinn, 2010: 7). Esta "descripción densa" (Espluga et al., 2010: 236) proporcionó conocimientos específicos de ciertas situaciones de relevancia e importancia social de las acciones subyacentes en torno a la Confianza y a los riesgos, peligros y amenazas basados en los discursos de los individuos y sus hogares que se conocen como "complex emergencies" (Coy, 2010: 22).

La definición de la población de estudio fue intencionada y tomó a los actores ligados a ambos conflictos socioambientales. La muestra de participantes en los grupos de discusión y entrevistas, así como su temporalidad se detallan en el Cuadro № 2.

Cuadro $N^{\circ} 2$

Matriz de Actores

\begin{tabular}{|l|l|l|l|}
\hline \multicolumn{1}{|c|}{ Técnica } & \multicolumn{1}{|c|}{ Actor } & \multicolumn{1}{|c|}{ Zona } & $\begin{array}{c}\text { Temporali- } \\
\text { dad }\end{array}$ \\
\hline Entrevista (2) & Políticos: & Castilla & mayo-junio \\
& -Diputados de la República de Chile, Distrito 5 & & \\
\hline
\end{tabular}


Continuación Cuadro $\mathrm{N}^{\circ} 2$

\begin{tabular}{|c|c|c|c|}
\hline Técnica & Actor & Zona & $\begin{array}{l}\text { Temporali- } \\
\text { dad }\end{array}$ \\
\hline Entrevista (3) & $\begin{array}{l}\text { Gobierno: } \\
\text { - Gobernador Provincia de Copiapó ( 2012-2013) } \\
\text { - Alcalde de Copiapó (1996-2008) } \\
\text { - Secretaría Regional Ministerial del Medio Ambien- } \\
\text { te, Región de Atacama }\end{array}$ & Castilla & mayo-junio \\
\hline Entrevista (2) & $\begin{array}{l}\text { Sociedad Civil: } \\
\text { - Administración Apostólica Copiapó. } \\
\text { - Miembro Mesa de Defensa Atacama. }\end{array}$ & Castilla & mayo-junio \\
\hline $\begin{array}{l}\text { Grupo de Dis- } \\
\text { cusión (3) }\end{array}$ & $\begin{array}{l}\text { Sociedad Civil: } \\
\text { - Comuna de Caldera (2); Pescadores artesanales, } \\
\text { dirigentes sociales, organizaciones ambientalistas. } \\
\text { Comuna de Copiapó (1); Dirigentes sociales, organi- } \\
\text { zaciones ambientalistas, universidades. }\end{array}$ & Castilla & mayo-junio \\
\hline Entrevista (2) & $\begin{array}{l}\text { Políticos: } \\
\text {-Senador circunscripción XVIII. } \\
\text {-Diputado de la República Chile, Distrito } 59 .\end{array}$ & Aysén & febrero-abril \\
\hline Entrevista (2) & $\begin{array}{l}\text { Gobierno: } \\
\text { - Alcalde Coyhaique (2013-2016) } \\
\text { - Secretaría Regional Ministerial Energía Aysén }\end{array}$ & Aysén & febrero-abril \\
\hline Entrevista (2) & $\begin{array}{l}\text { Sociedad Civil: } \\
\text { - Administración Apostólica de Aysén. } \\
\text { - Miembro Consejo defensa de La Patagonia }\end{array}$ & Aysén & febrero-abril \\
\hline Entrevista (1) & Empresa: HidroAysén & Aysén & febrero-abril \\
\hline $\begin{array}{l}\text { Grupo de Dis- } \\
\text { cusión (3) }\end{array}$ & $\begin{array}{l}\text { Sociedad Civil: } \\
\text { - Comuna de Coyhaique (2): Dirigentes sociales, } \\
\text { organizaciones ambientalistas, universidades. } \\
\text { - Comuna de Cochrane (1). Dirigentes sociales y } \\
\text { territoriales. }\end{array}$ & Aysén & febrero-abril \\
\hline
\end{tabular}

Fuente: Elaboración propia. 
El análisis de contenido tuvo directa relación con la percepción de la confianza, lo cual permitió conocer y analizar los mecanismos semiológicos y semánticos, es decir, el vínculo entre el contexto social y el individuo (Zinn, 2010). Las Entrevistas y Grupos de Discusión fueron grabadas (registro digital de audio) y posteriormente transcritas (formato word) y analizadas utilizando procedimientos basados en la Teoría Fundamentada, anclados en la comparación constante y el uso de paradigmas de codificación que garantizan el desarrollo conceptual y la densidad entre ambos casos (Glaser y Strauss, 1967). El análisis permitió elaborar una descripción, clasificación y conexión de los datos, contrastándolos de manera permanente y buscando "casos negativos para confirmar o desmentir las hipótesis, logrando así la triangulación de la información" (Boeck et al., 2006: 6), donde específicamente la técnica utilizada fue el "análisis temático cuantitativo" (Bardin, 1996).

Tras la codificación del discurso, se usó el software cualitativo Atlas-Ti 6.2, programa especializado que opera como un sistema de análisis de datos (CAQDAS: Computer Aided Qualitative Data Analysis System), que permitió una efectiva y transparente clasificación de los temas (Lores y Lozano, 2012).

\section{Análisis de las dimensiones conceptuales de la confianza en los proyectos socioambientales}

En términos generales, Castilla planteó un conflicto asociado no solo a una mala gestión de información difundida hacia la ciudadanía, sino que también a las inconsistencias en el comportamiento de la empresa y de las autoridades, además de la falta de veracidad de lo que la empresa informó, como se aprecia en la siguiente afirmación.

“A mí me tocó sentirme cómplice de lo que estaba ocurriendo cuando a nosotros nos vinieron a contar un cuento, como autoridades, de que iban a hacer un puerto y nosotros necesitábamos un puerto en la Región de Atacama y abrimos y vimos posibilidades de un puerto, cuando después sacaron bajo la manga, la situación de que lo que quería no era solamente un puerto, era la central termoeléctrica más grande de Sudamérica" (Grupo Discusión Copiapó).

En el relato del actor se grafica un panorama general y relevante de la percepción de la población en torno al déficit de confianza (credibilidad) depositada en la empresa y en las autoridades, pues las percepciones y discursos expresan que tanto autoridades públicas como privadas no mostraron un interés real de beneficiar a la población, sino que más bien de generar utilidades para sí mismos. Es decir, así como se desconfiaba de la empresa, la ciudadanía desconfiaba de las instituciones, organismos y personas a cargo de la evaluación y fiscalización del proyecto. Dicha desconfianza se expresaba en la poca credibilidad en las capacidades, competencias y habilidades de las personas en su rol de agente público, que debe velar por el bien común.

La primera dimensión general que aflora en la percepción que los actores tienen sobre las instituciones públicas como privadas es la "Benevolencia", entendida como la buena voluntad y la preocupación por el medio ambiente y la salud de la ciudadanía. El análisis releva, mayoritariamente, que el tema de los proyectos vinculados al medio ambiente requiere de una mayor inclusión ciudadana: "No es solo un tema de confianza o de desconfianza sino que es un tema de consideración de la ciudadanía" (Grupo Discusión Caldera), expresando que son las empresas ejecutoras de estos proyectos y las autoridades las que deben acercarse a la comunidad. En este sentido, existirían dos aristas en torno a la confianza que les inspiran las empresas “Una, es el comportamiento previo de las empresas en general y lo segundo también es el acercamiento de las empresas a la comunidad" (Mesa Defensa Atacama), donde la preocupación por la salud y el bien común son aspectos fundamentales para ellos, pues son quienes conviven a diario con los efectos y consecuencias que estos proyectos traen asociados.

Dado que existen evidencias de malos resultados en otras comunas, esto genera 
desconfianza frente a los nuevos proyectos, más aún en una región donde la temática ambiental es altamente relevante. Esto es validado por quienes mencionan que

\begin{abstract}
"Hay desconfianza frente a cualquier tipo de proyecto. Nosotros estamos en una Región donde estos temas son bastante sensibles" (Secretaría Regional Ministerial Medio Ambiente); "Hay desconfianza porque hay resultados malos en otros lados e incumplimiento y al final de cuentas quien se altera es la persona que localmente está ahí o que tiene algún rubro ahí" (Grupo Discusión Caldera).
\end{abstract}

Por otro lado, los participantes del estudio perciben la "integridad" como una dimensión clave, que relacionan con la honradez y transparencia de los actores públicos y privados. Tanto para los actores como para la ciudadanía, al existir un mayor flujo de información y, con ello, transparencia vinculada a un accionar honrado de las personas, existe una mayor posibilidad de ver íntegramente el problema al cual se enfrentan. Esta percepción se expresa en la siguiente afirmación: "Pero lo interesante acá es que cuando la información es más profunda y la gente se da cuenta de la verdadera razón de todo esto y alcanza a ver integradamente el tema" (Grupo Discusión Caldera).

El cumplimiento de los compromisos contraídos, que afectan a intereses ajenos, es otra de las dimensiones claves reveladas en los discursos de los actores, como se ejemplifica en la afirmación de un participante: "en Freirina a lo mejor el proyecto era viable, pero qué es lo que pasó, que la empresa presentó un proyecto, pero que después no cumplió lo que decía el proyecto y eso fue a lo mejor que tuvo que terminar y cerrar y que la gente, obviamente ... tenía toda la razón" (Grupo Discusión Caldera). Para la ciudadanía los compromisos que se establecen a través de los proyectos para las regiones y el país no suelen cumplirse, pues perciben que los beneficios de las instalaciones distan mucho de ir en favor de las localidades. Por el contrario, diversos participantes manifiestaron que los compromisos de las empresas e instituciones se orientan a sus propios intereses, ya que gran parte de la energía generada no queda en la región, ni siquiera en el país, sino que va al extranjero (en referencia a la venta de energía a Argentina): “El proyecto era (...) vender energía hacia Argentina, minera, ese proyecto Castilla, por eso tenía siete termoeléctricas y dos terminales de muelle, que se estimaban para los acopios" (Grupo Discusión Caldera).

Una de las variables objetivas en torno a la confianza que percibió la comunidad en Castilla fue "Competencia-Habilidad", expresando que

"En términos medioambientales las municipalidades tienen cero capacidad profesional, porque si nos dan una responsabilidad como esa debiera haber una dirección más que la dirección de medioambiente y que esté dotada de recursos, de los profesionales adecuados, de los profesionales que tengan los Conocimientos y la experiencia" (Ex Alcalde Copiapó).

Se requiere, por parte de los organismos/ instituciones, facultades y competencias necesarias para cumplir la función que se les determina (como las SEREMIS y no el Municipio). Se hace notar que esas competencias deben estar principalmente en aquellas encargadas del área medioambiental, las cuales son las evaluadoras -considerando que son ellas las que poseen mayor cantidad de profesionales con capacidades técnicas o legales- y poseedoras de la experiencia y los recursos necesarios (financieros, técnicos, humanos y de infraestructura) para ejecutar su función, como son el Sistema de Evaluación Ambiental y los organismos políticos como las Secretarías Regionales Ministeriales del Medio Ambiente y Salud. Lo anterior lo reafirma un participante expresando que "Los Municipios (...) no tienen la facultad en ciertos proyectos que desde el punto de vista medioambiental son amenazantes" (Ex Alcalde Copiapó). En este ámbito se destaca también la percepción de que no hay otras entidades públicas a las cuales la ciudadanía pueda consultar y confiar en sus respuestas ante la instalación de este tipo de proyectos y los impactos socioambientales que generan, manifestando que “¿Después del Municipio pa' arriba qué tiene como respaldo profesional? Nada. Nada. El municipio Ilega hasta la Dirección de Obra pa' arriba y no hay a quién consultarle si algo está bien o está mal" (Ex Alcalde Copiapó). 
La "Consistencia del comportamiento" es una dimensión que suele referirse a lo previsible de lo que se propone, y afecta tanto a la promesa como a los argumentos que se esgrimen. En torno a esta cuestión, son diversos los actores participantes que expresan que "Se dice que se hace, pero no se hace. Porque intervienen otros poderes que son fuertes" (Grupo Discusión Caldera). La falta de consistencia en el comportamiento se observó tanto en las empresas como en las instituciones. Las primeras, porque ofrecen beneficios para la comunidad con la finalidad de obtener aprobación de esta, pero finalmente no los cumplen Y, en el caso de las autoridades, porque en teoría tienen el deber de fiscalizar, pero, en la práctica, los ciudadanos perciben que las instituciones gubernamentales no fiscalizan o fiscalizan muy poco. En un sentido general, este hecho fue frecuentemente definido por los participantes como "negligencia", que se transforma en una debilidad del gobierno y de las entidades fiscalizadoras, pues se reconoce que: "Lo que hacía la empresa era citar autoridades, que era cambiar planes reguladores, que era comprar conciencia... que era comprar y comprar con plata" (Grupo Discusión Copiapó). Situaciones como esta, delatan la percepción pública de un comportamiento poco ético y negligente que pasaba por alto intereses socioambientales. Ante esto, la población se manifiesta frente a los proyectos que no son sustentables expresando que "Nadie está en contra de la minería, pero una minería de verdad sustentable, una minería que sea justa, una minería que genere riqueza para el país" (Grupo Discusión Copiapó).

Por último, en los textos analizados emerge el "Sentido de justicia", asociado a la "objetividad e imparcialidad". Esta variable se liga a que "La comunidad confíe en que los organismos estatales con competencia ambiental, van a hacer su pega, va a hacer la tarea que les está encomendada, que van a evaluar en forma profesional" (Secretaría Regional Ministerial Medio Ambiente), es decir, se confía en que los organismos estatales tienen las competencias para evaluar y fiscalizar un proyecto. Sin embargo, para que esto suceda es fundamental que quienes realicen dicha labor la efectúen de manera profesional, objetiva e imparcial, relevándose como una cuestión fundamental que el marco legal regule que las normas sean cumplidas efectivamente antes de construir y poner en funcionamiento el proyecto. En esta línea, uno de los participantes del estudio reclama:

"Tener un marco legal que sea de tal fortaleza que te exija ciertos cumplimientos que te aplique ciertas normas, que si tú cumples con esas leyes, con esas normas perfectamente el proyecto se puede construir" (Diputado de la República de Chile).

\section{El conflicto Hidroeléctrica Hidroaysén}

La visión general del conflicto desarroIlado en la Patagonia en torno al proyecto Hidroaysén plantea que la discusión está relacionada a los ámbitos de Benevolencia, de Cumplimiento de Compromisos y de la Consistencia del Comportamiento de los actores involucrados directa o indirectamente. En este sentido, se destaca que la confianza, como elemento central para la vinculación de la ciudadanía con la empresa y las autoridades (actores políticos), fue limitada debido a la percepción de la ciudadanía en cuanto a la inconsistencia en el comportamiento de las autoridades y su falta de compromiso. Dicha inconsistencia y falta de compromiso fue percibida no solo en torno a los actores, sino también hacia la ciudadanía, al no haberse respetado el derecho a la libertad de opinión.

La primera de las variables visibilizadas es la "Benevolencia", que se relaciona con la preocupación por el bien común y la buena voluntad. Esta dimensión emerge claramente en el análisis cuando se expresa que "La valentía que tiene el Seremi de Salud de rechazar el proyecto no la tuvieron los otros Seremis. El Seremi de Salud fue muy responsable al votar en contra del proyecto" (Alcalde Coyhaique). En esta misma línea se argumenta que es relevante que tanto la empresa como los actores políticos se relacionen con la comunidad de forma transparente y honesta. Uno de los actores expresa que "La relación con las comunidades no puede ser transaccional (...) que la relación de confianza tiene que ser transparente, honesta y no puede estar mediado por los recursos" (Empresa Hidroaysén). El discurso de los actores expresó que el proceso y conflicto generado en torno a Hidroaysén estuvo mediado o intervenido por recursos, primando los de tipo financiero. 
Este hecho, a la vista de los ciudadanos entrevistados no solo mermó la credibilidad del proyecto, sino también la confianza generada entre la empresa y los actores involucrados (ciudadanos y políticos)

"Eso es lo que falta, entonces eso produce tensión, porque no hay claridad, o sea la única claridad que hay los poderosos que hacen con la plata lo que quieren, eso es lo único" (Administración Apostólica Aysén).

La "Integridad", basada en la honradez y la transparencia, se alinea a lo planteado en el párrafo anterior, donde la percepción de los actores enfatiza en la importancia de esta expresando que "Se van generando mitos, se van generando leyendas, se va generando vacíos, se van generando dudas y al final cuando uno dice que es lo que se ha hecho, uno queda tan desconcertado como el primer día" (Grupo Discusión Cochrane). Por lo tanto, para los participantes la honradez y la transparencia son dos aspectos relevantes a la hora de comunicar información respecto a lo que se pretende hacer en una determinada localidad. La importancia de estos conceptos radica en que se asocian al concepto de integridad. Es decir, cuando se actúa bajo la honradez y la transparencia, es más fácil ver íntegramente el problema, sin intervención de factores que puedan estar limitando la visión objetiva sobre el tema. Así, el problema considera que en la gran mayoría de los proyectos medioambientales prima la postura de la empresa, pues su objetivo fue siempre obtener la aprobación y finalmente ejecutar el proyecto. Lo anterior es marcado por un actor que expone:

"Cada uno cuenta su verdad, la verdad que le conviene. HidroAysén ha hecho tremendas campañas de difusión, y ha tenido que estar ahí Patagonia Sin Represas diciendo que no todo lo que se está diciendo es tan cierto como lo pintan" (Alcalde Coyhaique).

El "cumplimiento de compromisos con los demás" relevado por los actores, es expresado en el siguiente relato:

"Misión objetivo de una empresa como HidroAysén es la utilidad po', o sea cuando, no sé si, no sé si han escuchado alguna vez a Daniel Fernández, a la María Irene Soto, o a Bernardo Larraín Matte o a Jorge Rossenburg, decir que este proyecto lo quieren llevar a delante porque es un buen negocio para sus accionistas" (Consejo Defensa de la Patagonia).

Aparentemente, no hay una observación definida y claramente expresada en relación al cumplimiento de compromisos establecidos por la empresa ejecutora del proyecto. Sin embargo, se observó una visión específica de algunos participantes que reconocen la existencia de fines particulares tras la ejecución del proyecto, intereses que no van en favor de la comunidad, sino que son netamente económicos: "toda decisión que tome el ser humano, si está basado en la codicia o es más moral la codicia, hablemos de la acumulación, acumulación fundamentalmente económica" (Consejo Defensa de la Patagonia).

La "Consistencia del comportamiento" impacta en Hidroyasén cuando un actor expone: "Le quisieron quemar la casa a un compadre porque pensaba que el proyecto era bueno, a otros los han insultado, a otros les han quebrado los vidrios de sus vehículos o sea por esas cosas, nadie se arriesga a decir hoy en día a decir, estoy a favor del proyecto" (Grupo Discusión Cochrane). Lo que emerge de la sentencia anterior es un escenario radicalizado por el comportamiento y las acciones que toman quienes no son partidarios del proyecto contra quienes consideran que el proyecto es beneficioso. Las acciones violentistas intimidaron a parte de la ciudadanía, que decidió no manifestar abiertamente su posición sobre el proyecto. Esta situación potenció que, en los medios de comunicación, se mostraran con más frecuencia posturas en contra que a favor del proyecto.

Otro aspecto central a destacar es la percepción de inconsistencia en el comportamiento de las personas que se manifestaban en contra del proyecto HidroAysén en la Patagonia, debido a que, aperentemente, decían una cosa a la comunidad, pero acababan haciendo otra. El siguiente fragmento reseña lo anterior:

“El alcalde de Tortel, Bernardo López, ... dice proteger la Patagonia, proteger Tortel y sus alrededores pero resulta que él es un personaje bastante contradictorio porque tiene un proyecto donde vende agua milenaria y no es chiste, el protector 
de la Patagonia vendió en favor a un empresario norteamericano" (Diputado de la República de Chile).

La objetividad e imparcialidad dada por la variable "Sentido de justicia", es expresada por los actores cuando se expone que: "Para el resguardo de la población y para los beneficios, hoy día no puede llegar cualquier proyecto a un territorio y no comprometerse con el territorio" (Grupo Discusión Cochrane). Así, en el discurso de los participantes se destacó también que es necesario que las empresas que llegan a instalarse en un territorio específico se comprometan con el territorio, puesto que los recursos que son explotados son de la comunidad y no de derecho puramente privado. Un participante describe este sentimiento diciendo que "las riquezas tienen que ser para todos no solo para algunos 'noma' como sucede hoy día" (Diputado de la República de Chile) lo cual enfatiza por parte de la ciudadanía el sentido de justicia que debieran traer este tipo de proyectos, pues

"La ciudadanía reclama, y con justa razón. Y ¿por qué reclama? Porque aparte de hacer un daño sabemos que hay mejores alternativas, o sea hoy en día uno evalúa pequeñas, medianas centrales, centrales de pasada, eólicas, solares, son más baratas, entonces ¿pa' qué?, entonces en el fondo es sostener un negocio y un modelo" (Senador de la República de Chile).

Por último, la variable objetiva "Competencia-Habilidad" es importante para los actores, cuando se expresa que "Es casi imposible revisar 1.500 hojas de un proyecto como HidroAysén, imposible" (Grupo Discusión Cochrane).
Los actores locales consideraron de vital importancia que quienes debían evaluar este tipo de proyectos poseyeran las competencias o habilidades necesarias. En las narrativas analizadas se identifica que hubo problemas desde la fase de presentación de los proyectos (inicio), aduciendo que el tiempo disponible para los técnicos no era suficiente para efectuar una evaluación correcta y eficaz. En este sentido, se destaca que aunque se cuente con recursos humanos y técnicos con experiencia, los encargados de esta labor no logran Ilevarla a cabo pues sus capacidades se ven sobrepasadas: "No le bastaban 30 días para tomar una apreciación informativa a ellos no les alcanza, ingenieros todas estas áreas, quedaban con un vacío impresionante" (Grupo Discusión Cochrane). En el mismo sentido, un participante identificó que es necesario mejorar los mecanismos de seguimiento de los proyectos, mencionando que "Si hay un proyecto monstruoso también habrá que implementar un seguimiento, una estructura superior a la capacidad que tiene la región" (Administración Apostólica Aysén). En su opinión las instancias y los organismos locales-regionales pueden estar limitados en cuanto a recursos y capacidades para el seguimiento y fiscalización de los procesos de ejecución de proyectos de grandes magnitudes como es el caso de HidroAysén.

El análisis realizado para los conflictos Termoeléctrica Castilla e Hidroeléctrica $\mathrm{Hi}$ droyasén nos permiten conceptualizar lo trabajado a través del siguien mapa conceptual, el cual funge como una malla que grafica y sentitiza lo encontrado en la investigación (Figura $\mathrm{N}^{\circ} 1$ ). 
Figura $N^{\circ} 1$

Malla de Implicación dimensiones conceptuales de la confianza

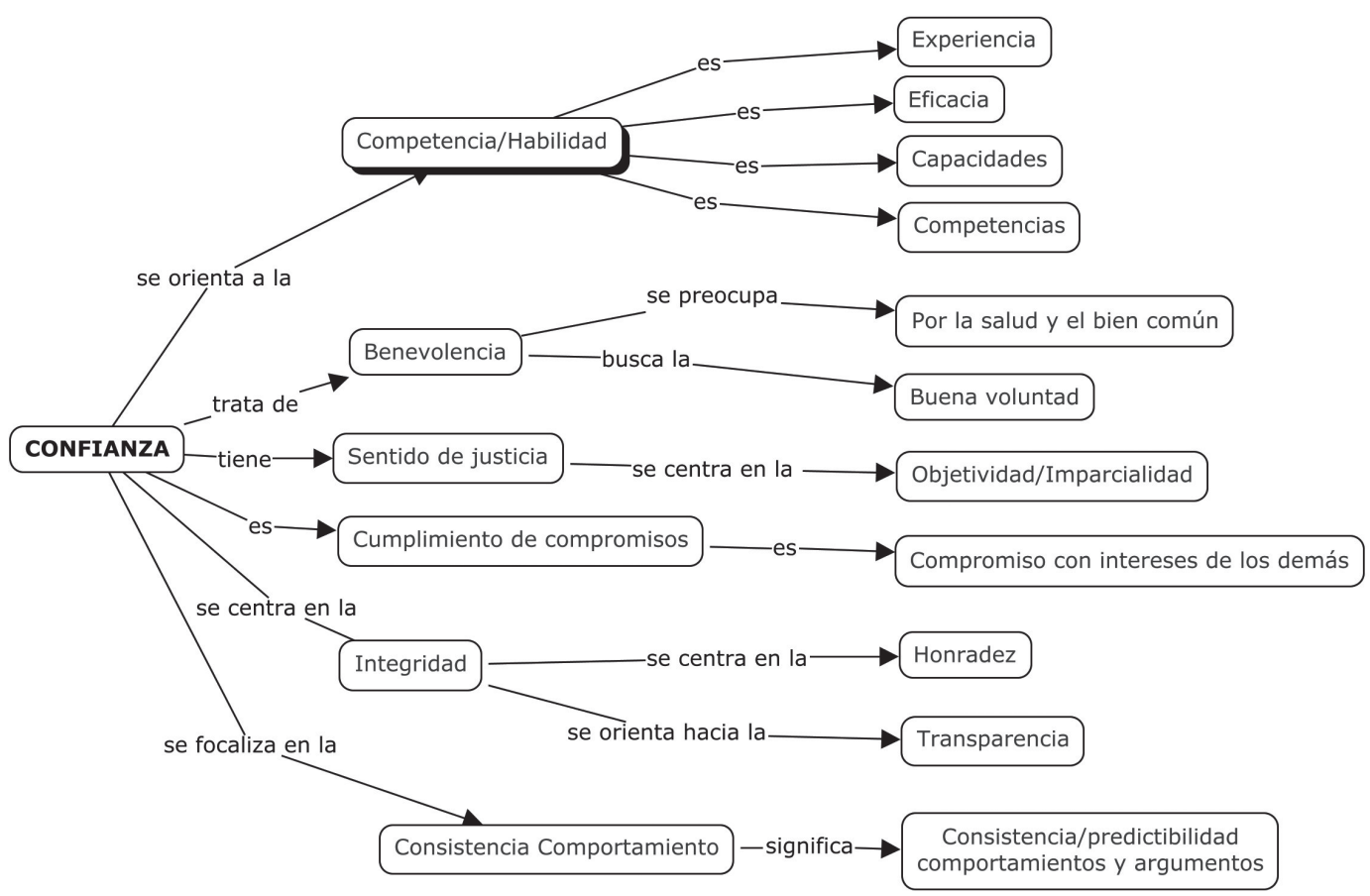

Fuente: Elaboración propia.

\section{Conclusiones}

El análisis realizado revela la centralidad de la variable "Competencia-Habilidad". En este sentido, los actores expresan la necesidad de competencias técnicas por parte de las instituciones evaluadoras y de las personas que realizan labores de evaluación y fiscalización. En referencia a Castilla, la dimensión competencia-habilidad aparece en la percepción de una carencia de profesionales e instituciones a nivel local con las capacidades, experiencia y recursos necesarios para fiscalizar el proyecto y dimensionar sus alcances. En el caso de Hidroaysén, esta variable concierne a las limitaciones para evaluar de manera eficaz que aparentemente tienen los profesionales a cargo de las áreas de medio ambiente, ya que las exigencias sobrepasaron sus capacidades logísticas.

La segunda variable que se relevó en el análisis presentado es la "Consistencia en el comportamiento". En ambos conflictos, se percibe una inconsistencia de comportamiento en los principales actores involucrados en el proceso. Sin embargo, hay una diferencia sustancial entre ambos casos. Quienes han vivido el conflicto del proyecto Castilla percibieron que existió una falta de consistencia en el comportamiento de la empresa y de las autoridades de la comuna. Los participantes interpretaron que la empresa ofreció beneficios solamente para obtener la aprobación ciudadana, pero no informó a la comunidad de los pormenores del proyecto. También percibieron que las autoridades no fiscalizaron el proceso debidamente y afirmaron que se dieron relaciones de clientelismo con la empresa. Por otra parte, en el proyecto Hidroaysén hubo una percepción de falta de consistencia en el comportamiento de los actores que se manifestaban en contra del proyecto, quienes aparentemente defendían su postura e ideales pasando por alto la libertad de expresión, en contra de quienes apoyaron el proyecto, amedrentándolos y amenzándolos de dañar su propiedad privada. Ante esta situación, algunos participantes denunciaron que hubo autoridades que comunicaron una posición a la población, cuando, en su vida privada, mantenían negocios con recursos naturales. 
En la dimensión afectiva o subjetiva, "la benevolencia" emergió como una variable destacable. Tanto para Castilla como $\mathrm{Hi}$ droaysén se desarrolló un sentimiento de preocupación por el bien común y por los daños que estos proyectos podían generar a la comunidad, no solo desde una perspectiva medioambiental, sino también abordando los potenciales problemas de la salud de quienes convivirían cotidianamente con las instalaciones energéticas.

Otra variable de la dimensión afectiva mencionada y relevada como importante por los actores del estudio fue la "Integridad". Tanto en Castilla como HidroAysén es posible señalar que a la población le fue imposible conocer realmente el conflicto en el cual estaban insertos, pues ante la falta de honradez de las empresas, quienes presentaban a la población las áreas favorables de los proyectos, se dejaban de lado los costes y riesgos asociados. Ante esta situación, fue la ciudadanía quien a partir de la búsqueda y difusión de información alternativa, comunicara aspectos alusivos a los proyectos para ampliar el panorama y la visión de los acontecimientos en sus localidades, poniendo a disposición de todo el público información que les inspirara confianza y conocimiento del tema.

El Sentido de "Justicia" también cobró relevancia en el estudio por los actores ligados a ambos conflictos. En Castilla las personas orientaron su percepción del sentido de justicia hacia la necesidad de confianza en los organismos estatales fiscalizadores, para que desde su visión objetiva e imparcial efectuasen las evaluaciones necesarias. En cambio, en Hidroaysén se orientó la visión del sentido de justicia hacia la manifestación ciudadana, quienes desde una posición objetiva podían contribuir a proporcionar una perspectiva que abriera el debate, pues la ciudadanía sería más imparcial y no perseguiría fines particulares económicos.

Por último, respecto al "cumplimiento de compromisos", se observó que tanto en Castilla como HidroAysén existió una amplia incertidumbre, pues nadie les garantizó que una vez aprobado el proyecto, las empresas seguirían cumpliendo lo propuesto a las personas o a la comunidad en general. Se percibió que en numerosas ocasiones los acuerdos fueron de palabra, quedando en la memoria colectiva la idea de que las empresas otorgaron de palabra propuestas y beneficios solo para conseguir la aprobación de la ciudadanía. De esta forma la ciudadanía se transformó, ante la visión empresarial, en la piedra de tope para la ejecución del proyecto.

Los efectos de la confianza sobre la aceptación de riesgos deben ser matizados caso a caso, pues se ven permanentemente condicionados por aspectos contextuales. Teniendo esto en cuenta, los resultados de la investigación señalan que la confianza es una dimensión relevante a la hora de dar cuenta de los riesgos y peligros asociados a conflictos socioambientales por energía en Chile. Las probabilidades de decepción son mayores si este tipo de dimensiones sociales no son tomadas en cuenta. Por lo tanto, se hace necesario que los diseños de intervención socioambiental incorporen tales dimensiones en pos de una mejor gobernanza de una sociedad moderna que produce y reproduce problemas y conflictos de compleja solución.

\section{Referencias bibliográficas}

AA.VV. Escenarios energéticos Chile 2030. Construyendo escenarios y desafiando paradigmas. 2010. Disponible en Internet: http://www.escenariosenergeticos. cl/wp/wp-content/uploads/2010/08/Escenarios-Energ\%C3\%A9ticos_Chile-20301.pdf

AGUILAR, C. y VALENZUELA, R. Crisis energética. Propuestas científicas en energías renovables no convencionales en Chile entre los años 2000 y 2010. Temuco: Tesis de Licenciatura, Universidad de La Frontera, 2010

ALTIERI, M. y ROJAS, A. La tragedia ecológica del milagro neoliberal chileno. Persona y Sociedad, Vol. XII, No 1, 1999, p. 127-141.

BARDIN, L. El Análisis de Contenido. Madrid: Ediciones Akal, 1996.

BECK, U. La sociedad del riesgo mundial. En busca de la seguridad perdida. Barcelona: Paidós, 2008 
BECK, U. La Sociedad del Riesgo. Hacia una Nueva Modernidad. Barcelona: Paidós, 2006.

BECK, U. La Sociedad del Riesgo Global. Madrid: Siglo XXI, 2002.

BECK, U. Ecological Enlightment. Nueva Jersey: Humanity Press, 1995

BOECK, T.; FLEMING, J. \& KEMSHALL, H. The Context of Risk Decisions: Does Social Capital Make a Difference? In: Forum Qualitative Sozialforschung/Forum: Qualitative Social Research, 2006, Vol. 7, № 1. Disponible en Internet: http://www.qualitative-research.net/ index.php/fqs/article/view/55/113

BULLOCH, S. Seeking Construct Validity in Interpersonal Trust Research: A Proposal on Linking Theory and Survey Measures. Social Indicators Research, 2013, Vol. 113, № 3, p. 1289-2310.

CAMUS, P. y HAJEK, E. Historia Ambiental de Chile. Santiago de Chile: Ediciones Pontificia Universidad Católica de Chile, 1998.

CÁRCAMO, P.; CORTÉS, M.; ORTEGA, L.; SQUEO, F. y GAYMER, C. Crónica de un conflicto anunciado: Tres centrales termoeléctricas a carbón en un hotspot de biodiversidad de importancia mundial. Revista Chilena de Historia Natural, 2011, No 84, p. 171.-180.

CHRYSSOCHOIDIS, G.; STRADA, A. \& KRYSTALLIS, A. Public trust in institutions and information sources regarding risk management and communication: towards integrating extant knowledge. Journal of Risk Research, 2009, Vol. 12, No 2, p.137-185.

COY, M. Los estudios del riesgo y de la vulnerabilidad desde la geografía humana. Su Relevancia Para América Latina. Población y Sociedad, 2010, No 17, p. 9-28.

DOUGLAS, M. Risk Acceptability according to Social Science. New York: Basics book, 1986.

EISIER, J.; STAFFORD, T.; HENNEBERRY, J. \& CATNEY, P. Trust me, I'm a scientist (not a developer): Perceived expertise and motives as predictors of trust in assessment of risk from contaminated land. Risk Analysis, 2009, Vol. 29, No 2, p. 288-297.

EL MOSTRADOR. El New York Times irrumpe ante aprobación de HidroaAysén y lo califica como un error irreparable, 2011. Disponible en Internet: http://www.elmostrador.cl/noticias/ pais/2011/05/24/sacrificar-la-patagonia-por-elpoder-seriaun-error-irreparable

ESPLUGA, J.; PRADES, A. \& GONZALO, J. Comunicating at the edge: Risk Communication Processes and Structural conflicts in highly industrialized petrochemical areas. Catalan Journal of communication\& Cultural Studies, 2010, Vol. 2, № 2, p. 231-251.

ESPLUGA, J.; PRADES, A.; GAMERO, N. y SOLA. El papel de la confianza en los conflictos socioambientales. Política y Sociedad, 2009, Vol. 46, No 1-2, p. 255-273.

ESPLUGA, J. Conflictes socioambientals i l'estudi de la percepció social del risc. Papers, 2004, No 72, p. 145-162.

ESPLUGA, J. Per una sociología dels riscos tecnològics. Ambits de Politítica i Societat, 2007, No 36, p. 34-38.

ESPLUGA, J. y FARRE, J. Más allá de la percepción: la comunicación del riesgo. In: FARRE, J. \& FERNANDEA, J. Comunicació $i$ risc petroquímic a Terragona. De les definicions a les pràctiques institucionals. Terragona: Publicaciones URV, 2007.

FISKE, E. Reconceptualizing Environmental Conflict Resolution: The Development Facilitation Approach. In: Ponencia presentada a la conferencia Alternative Dispute Resolution and Natural Resources: Building Consensus and Resolving Conficts in the Twenty-first-Century. Tucson, 2000.

GIDDENS, A. Las consecuencias de la modernidad. Madrid: Alianza, 1993.

GIRO PAIS. Qué implica la Termoeléctrica Castilla, 2010. Disponible en Internet: http://www.giropais.cl/content/view/1410869/ Queimplica-la-Termoelectrica-Castilla.html

GLASER, B. \& STRAUSS, A. The Discovery of Grounded Theory: Strategies for Qualitative 
Research. New York: Aldine Publishing Company, 1967.

KASPERSON, R.; GOLDING, D. \& TULER, S. Social distrust as a factor in siting hazardous facilities and communicating risks. Journal of social issues, 1992, Vol. 48, $\mathrm{N}^{\circ} 4, \mathrm{p}$. 161-187.

KNIGHT, F. Risk, Uncertainty and Profit. Orlando: Signalman Publishing, 2013 [1921].

LORES, M. y LOZANO, N. Percepciones y usos de los Social Media en la comunicación de riesgos y beneficios alimentarios: Ios consumidores europeos como caso de estudio. En: III Congreso AE IC: Comunicación y Riesgo, Tarragona, 2012.

LUHMANN, N. La sociedad de la sociedad. México: Herder/Universidad Iberoamericana, 2007.

LUHMANN, N. Sociología del Riesgo. México: Universidad Iberoamericana, 2006.

LUHMANN, N. Confianza. Barcelona: Anthropos, 1996.

MAIRAL,; G. La década del riesgo. Situaciones y narrativas de riesgo en España a comienzos del siglo XXI. Madrid: Editorial Catarata, 2013.

MAJLUF, N.; ABARCA, N. y MINGO, S. La Confianza en la Empresa. Santiago de Chile: Documento de Trabajo, Departamento de Ingeniería Industrial, Pontificia Universidad Católica de Chile, 2003.

NEW YORK TIMES, Keep Chileans Patagonia Wild. 2012. Disponible en Internet: http://www.nytimes.com/2011/05/24/ opinion/24tue3.html?_r=4\&scp=1\&sq=patago nia+chile\&st=cse Visita:

OCDE. Evaluaciones del desempeño ambiental. Santiago de Chile: CEPAL, 2005.

OLTRA, C.; BOSO, A. \& PRADES, A. Challenges in the research of public acceptance of energy technologies, infrastructures and applications. Madrid: Ciemat, 2014. Disponible en Internet: http://documenta.ciemat.es/ handle/123456789/133
O'MALLEY, P. Risk, Uncertainty and Government. London: Cavendish Press/Glass-House, 2005.

O'MALLEY, P. (editor). Governing Risks. Aldershot: Ashgate, 2002.

PETERS, R.G.; COVELLO, V.T. \& McCALLUM, D.B. The determinants of trust and credibility in environmental risk communication: An empirical study. Risk analysis, 1997, Vol. 17, No 1, p. 43-54.

PIGDEON, N.; HOOD, C.; JONES, D. \& TURNE, B. Risk perception. The Royal Society Risk: Analysis, Perception and Management. London: Report of a Royal Society Study Group, 1992, p. 89-134.

POORTINGA, W. \& PIGDEON, N. Exploring the dimensionality of trust in risk regulation. Risk analysis, 2003, Vol. 23, No 5, p. 961-972.

QUIROGA, R. La sustentabilidad socioambiental de la emergente economía chilena entre 1974 y 1999. Evidencias y desafíos. En: SADER, E. (compilador). El ajuste estructural en América Latina. Costos sociales y alternativas. Buenos Aires: CLACSO, 2001.

REEN, O. \& LEVINE, D. Credibility and trust in risk communication. En: KASPERSON, R.E. \& STALLEN, P. (editors). Communicating risk to the public. Dordrech: Kluwer, 1991, p. 175-218.

RODRÍGUEZ, D. y MAJLUF, N. La Confianza en Chile: Sus dificultades y posibilidades. Santiago de Chile: Documento de Trabajo, Departamento de Ingeniería Industrial, Pontificia Universidad Católica de Chile, 2003.

RODRÍGUEZ, H. La confianza pública en las instituciones reguladoras de riesgo: tres modelos de confianza para tres desafíos del análisis del riesgo. Argumentos de Razón Técnica, 2009, $\mathrm{N}^{\mathrm{o}} 12$, p. $125-153$.

ROMERO-TOLEDO, H. Ecología política y represas: elementos para el análisis del Proyecto HidroAysén en la Patagonia chilena. Revista de Geografía Norte Grande, 2014, № 57, p. 161-175. 
ROMERO-TOLEDO, H.; ROMEROARAVENA, H. y TOLEDO, X. Agua, Poder y Discursos: Conflictos Socio-territoriales por la construcción de centrales hidroeléctricas en la Patagonia Chilena. Anuario de Estudios Americanos, 2009, Vol. 66, № 2, p. 81-103.

ROMERO-TOLEDO, H.; MÉNDEZ, M.; SMITH, P. y MENDOZA, M. Enfoque EcológicoSocial de la variabilidad climática, extracciones de agua y demandas territoriales en las cuencas del Desierto de Atacama. Geonorte, 2012, № 4, p. 261-287.

ROJAS, A.; SABATINI, F. y SEPÚLVEDA, C. Conflictos ambientales en Chile: aprendizajes y desafíos. Ambiente y Desarrollo, 2003, Vol. XIX, $\mathrm{N}^{0} 2$, p. 22-30.

ROSA, E.; RENN, O. \& MCCRIGHT, A. The risk society revisited. Social Theory and Govenance. Pennsylvania: Temple University Press, 2013.

SMITH, N.; CEBULLA, A.; COX, L. \& DAVIES, A. Risk Perception and the Presentation of Self: Reflections from Fieldwork on Risk. Forum Qualitative Sozialforschung, 2006, Vol. 7, № 1. Disponible en Internet:

http://www.qualitative-research.net/index. php/fqs/article/view/54

SEPÚLVEDA, C. y ROJAS, A. Conflictos Ambientales y reforma ambiental en Chile: una oportunidad desaprovechada. Ambiente y Desarrollo, 2010, Vol. XXIV, No 2, p. 15-23

SEPÚLVEDA, C. y GEISSE, G. El caso de Golden Spring: la construcción social de la demanda ambiental entre los habitantes de Compu. Ambiente y Desarrollo, 1995, Vol. XI, № 1, p. 59-66.

SOLA, R.; PRADES, A.; ESPLUGA, J. y REAL, M. Confianza, Incertidumbre y Percepción Social de las Tecnologías Avanzadas. Un estudio de caso. Revista Internacional de Sociología, 2009, No 67, p. 161-175.

TEJEDA, N. y PÉREZ-FLORIANO, L. La amplificación social de los riesgos: evidencias del accidente en la mina Pasta de Conchos. Comunicación y Sociedad, 2011, № 15, p. 71-99.
TERWEL, B.W.; HARINCK, F.; ELLEMERS, N. \& DAAMEN, D.D. Competence Based and Integrity Based Trust as Predictors of Acceptance of Carbon Dioxide Capture and Storage (CCS). Risk Analysis, 2009, Vol. 29, No 8, p. 1.129-1.140.

UPHAM, P.; OLTRA, C. \& BOSO, À. Towards a cross-paradigmatic framework of the social acceptance of energy systems. Energy Research and Social Science, 2015, No 8, p. 100-112.

VALLEJOS-ROMERO, A.; SALVO, S. y GARRIDO, J. Las dimensiones sociales de los riesgos en torno a conflictos socioambientales por energía en Chile: la confianza en el conflicto "Termoeléctrica Castilla". Papers, 2015 (en prensa).

VALLEJOS, A. La relevancia de la confianza institucional y la comunicación en la percepción y construcción social de riesgos. Perfiles Latinoamericanos, 2012, Nº 39, p. 151-176.

VALLEJOS, A. Conflictos Ambientales en una Sociedad Mundializada. Algunos antecedentes y consideraciones para Chile. Revista LIDER, 2005, No 13, p. 193-214.

VILCHES, C. Vilches (UDI) defiende que se instale termoeléctrica Castilla en zona donde tiene intereses mineros. El Mostrador, 2010. Disponible en Internet:

http://www.elmostrador.cl/noticias/ pais/2010/11/16/vilches-udi-dice-que-dictamen-determoelectrica-castilla-debe-ser-corregido-y-permitir-instalacion/

VISSCHERS, V.H.; KELLER, C. \& SIEGRIST, M. Climate change benefits and energy supply benefits as determinants of acceptance of nuclear power stations: Investigating an explanatory model. Energy policy, 2011, Vol. 39, № 6, p. 3.621-3.629.

ZINN, J. Biography, Risk and Uncertainty-Is there Common Ground for Biographical Research and Risk Research? Journal Forum Qualitative Social Research, 2010, Vol. 11, No 1, p. 1-15.

ZINN, J. (editor). Social Theories of Risk and Uncertainty. An Introduction. Oxford: Blackwell Publishing, 2008. 
